\title{
Pre-operative Medical Assessment and Optimisation
}

\author{
Helen Wilson and Amy Mayor
}

\subsection{Pre-operative Medical Assessment}

Comprehensive Geriatric Assessment (CGA) has a strong evidence base in reducing mortality, increasing the number of patients discharged back to their own homes and reducing length of stay [1]. This approach should form the basis of assessment for any older person with frailty in hospital.

The pre-operative medical assessment forms part of an interdisciplinary review to understand the medical conditions that a patient may have in the context of their functional ability. The medical history can be evaluated by physicians, anaesthetists, peri-operative physicians, orthogeriatricians, or any frailty practitioner with skills in developing a clear understanding of the implications of co-morbidities on an individual's ability to function in addition to gauging the likely impact of trauma, anaesthesia and surgery.

In addition, therapists often conduct initial assessments to gather information about mobility, activities of daily living, cognition, mood, environmental and social circumstances.

This chapter is a component of Part 2: Pillar I.

For an explanation of the grouping of chapters in this book, please see Chapter 1: 'The Multidisciplinary Approach to Fragility Fractures Around the World-An Overview'.

\section{H. Wilson $(\bowtie)$}

Royal Surrey County Hospital, Guildford, UK

e-mail: hwilson6@nhs.net

\author{
A. Mayor \\ Calderdale and Huddersfield Hospital, Huddersfield, UK \\ e-mail: Amy.mayor@cht.nhs.uk
}




\subsection{Information Gathering}

This can be more complex than it seems with older patients often unaware of their personal medical history, previous investigations or reasons for prescribed medications. A significant proportion also has cognitive impairment and is unable to provide information. Collateral history from carers, the primary care physician, previous hospital medical notes, previous imaging and pathology results are key to piecing together a complete picture.

Having a standardised clerking proforma can help to ensure that all necessary information is captured, including a pre-operative assessment of cognition. This together with collateral from family/friends/carers can identify those with established dementia in addition to those with likely undiagnosed dementia. These patients are at particularly high risk of developing peri-operative delirium. Proactive orthogeriatric management has been shown to reduce the incidence of delirium after hip fracture by one-third and severe delirium by a half [2].

Studies have looked at using haloperidol routinely peri-operatively in those at risk of delirium. A randomised placebo-controlled trial of 430 patients given either placebo or haloperidol $1.5 \mathrm{mg} /$ day showed no reduction in incidence of delirium, but it did reduce the severity and duration of delirium with a reduction in length of stay. Its routine use is not recommended [3].

The 4AT is a useful tool for recognising and monitoring delirium [4]. It is a simple score which can be performed with good reliability by all staff and requires no specific training. It has been validated in patients with hip fracture [5] and should be a routine part of hip fracture management.

A description of an individual's functional ability adds to an understanding of the impact and severity of co-morbidities, particularly with regard to cardiac and respiratory disease. This is often described in metabolic equivalents (METS) with one MET being defined as the amount of oxygen consumed while sitting at rest and is equal to $3.5 \mathrm{~mL} \mathrm{O}_{2} / \mathrm{kg} / \mathrm{min}$ [6]. Those able to undertake activity such as easily managing a flight of stairs (four METs or more) are unlikely to have significant cardiorespiratory disease and have low cardiovascular risk (see Table 7.1).

Table 7.1 Metabolic equivalents

\begin{tabular}{|l|l|}
\hline Physical activity & METs \\
\hline Sitting reading/watching television & 1.0 \\
\hline Washing and dressing independently & 2.1 \\
\hline Walking slowly on flat & 2.3 \\
\hline Gentle household activity, e.g., cooking/cleaning & 2.5 \\
\hline Walking a small dog (3 km/h) & 2.7 \\
\hline Light static cycling/bowling & 3.0 \\
\hline Gardening or outdoor activity & 3.6 \\
\hline Walking quickly $(5 \mathrm{~km} / \mathrm{h})$ & 3.6 \\
\hline Climb flight of stairs without stopping & 4.0 \\
\hline Dancing & 4.5 \\
\hline Playing tennis/racquet sports & 8.5
\end{tabular}


Those with low levels of activity may have asymptomatic underlying cardiovascular disease or may be limited by musculoskeletal disorders including arthritis, osteoporosis with kyphosis, sarcopenia or indeed obesity.

\subsection{Cardiovascular Disease}

Patients with a history of ischaemic heart disease are clearly at risk of peri-operative cardiac events. Cardiovascular risk factors should also be considered including the presence of diabetes, hypertension and smoking.

The Goldman cardiac risk index [7] or the Revised Cardiac risk index [8] may be used to identify high-risk patients and predict the likelihood of peri-operative cardiac event or death.

A baseline electrocardiogram may give indications of asymptomatic cardiac disease with left bundle branch block or evidence of q waves or poor r-wave progression in the anterior leads.

An echocardiogram will give an indication of regional wall abnormalities from myocardial infarction, an estimate of left ventricular function and an indication of underlying valvular heart disease. This information can assist with risk stratification but should not delay surgery.

Patients with suspected coronary artery disease should be discussed with an anaesthetist. Unless a patient is symptomatic with cardiac chest pain, surgery should not be delayed to perform cardiac investigations. Routine troponin measurements are not helpful and do not correlate with early mortality [9]. Those already on beta blockers should continue their usual dose pre-operatively unless there is significant bradycardia or hypotension. Attention to haemoglobin levels is important, as perioperative anaemia may increase cardiac strain and increase the risk of a cardiac event.

\subsubsection{Valvular Heart Disease}

Cardiac murmurs are often present in older people, with insignificant aortic sclerosis or mild mitral regurgitation being the most common. A large retrospective study showed that $6.9 \%$ of patients with hip fracture had previously undiagnosed significant aortic stenosis [10]. This may influence the type of anaesthetic and the need for invasive cardiac monitoring. Significant aortic stenosis is suspected if the patient has an ejection systolic murmur in the aortic area in combination with a history of angina on exertion, unexplained syncope or near syncope, a slow rising pulse clinically in the brachial artery and an absent second heart sound or LVH on the ECG without hypertension. Patients with significant aortic stenosis require careful fluid balance and are at high risk of pulmonary oedema. Echocardiography should not delay timing of operation but may be useful if readily available. 


\subsubsection{Heart Failure}

Many older patients will have a history or symptoms in keeping with poor ventricular function on a background of hypertension, ischaemic heart disease, valvular heart disease or atrial fibrillation. The mainstays of medical treatment are diuretics, ACE inhibitors, angiotensin receptor blockers, beta blockers, aldosterone antagonists and a combination of hydralazine and nitrates. Increasingly, therapies for heart failure include electrophysiological interventions such as cardiac resynchronisation therapy (CRT), pacemakers with or without implantable cardioverter-defibrillators (ICDs). A recent echocardiogram can be useful to evaluate the severity of left ventricular dysfunction but should not delay operation. Severity can usually be gauged from the history, symptoms and required medication.

Those who are euvolaemic should undergo early surgery, omitting heart failure medication until $48-72 \mathrm{~h}$ post-operatively to reduce the incidence of symptomatic low blood pressure preventing mobilisation. Caution should be observed with administering intravenous fluid. Anaemia should be managed proactively to maintain haemoglobin levels above $100 \mathrm{~g} / \mathrm{L}$. Once able to transfer out of bed medication can be slowly re-introduced. These patients often develop increasing peripheral oedema 5-7 days post-operatively and may require an increased dose of diuretics for a period of time.

Patients with decompensated heart failure and fluid overload at presentation need careful attention. Those with acute left ventricular failure need stabilising before theatre. This is often associated with an acute ischaemic event. Anti-platelet and anti-coagulant therapy may cause increased blood loss at the fracture site and should only be started with caution for acute cardiac ischaemia. Discussion with cardiologists regarding appropriate intervention and an individualised decision about timing of surgery should be made.

Those with poor right ventricular function and fluid overload need high-dose diuretics with close monitoring of peripheral oedema, weight and renal function. This is often associated with hyponatraemia, hypotension and renal impairment and requires close observation. Correction to achieve a euvolaemic state often takes 5-10 days. It is usually better to proceed with surgery and manage the decompensated heart failure in the post-operative period. Significant peripheral oedema in the thigh however may increase the risk of wound breakdown.

\subsubsection{Conduction Defects, Pacemakers and Implantable Cardiac Defibrillators (ICD)}

Conduction defects seen on the 12-lead ECG are very common in older people. Temporary pacing is only indicated if a patient has complete heart block or has had syncope related to tri-fascicular heart block. First-degree heart block, bundle branch block and ectopics are of unlikely significance if asymptomatic and do not require pre-operative investigation. 
Pacemakers have become increasingly sophisticated, and a basic knowledge of different devices and their indications is required to aid the acute management of patients with fragility fracture. All patients with pacemakers have routine annual checks, and a pre-operative check is only required if there is concern about malfunction or if it has not been checked within 12 months.

It is important to understand the reason for the device and whether the patient is pacemaker dependent. External pacing equipment and a defibrillator must be available during surgery. The use of surgical diathermy/electrocautery can give rise to electrical interference, and this can present additional risks when used in patients with pacemakers and ICDs. Energy can also be induced in heart lead systems causing tissue heating at lead tips through high-frequency current [11]. The manufacturers recommend avoiding surgical diathermy if surgery is occurring within $50 \mathrm{~cm}$ from the device. If diathermy is deemed essential, then the use of bipolar diathermy with short bursts of energy minimises the risk. Where available, the use of a harmonic scalpel should be considered.

If a cardiac technician is available, then an ICD can be turned onto monitor only mode to prevent shock delivery during surgery. Otherwise ICDs should be turned off by placing a magnet over the device which should be secured with micropore tape. Any sustained ventricular tachycardia or ventricular fibrillation intraoperatively should be managed with external defibrillation. Post-operatively, the magnet should be removed, and the patient monitored until the device has been checked.

\subsubsection{Atrial Fibrillation (AF)}

Public campaigns such as 'know your pulse' have increased public awareness of the risk of stroke from atrial fibrillation. Patients with permanent AF and a controlled ventricular rate should continue with rate control medication (usually a betablocker, dioxin or verapamil) pre-operatively, with their usual dose administered on the day of surgery. Some patients are known to have paroxysmal atrial fibrillation (PAF). Amiodarone, flecainide or beta blockers are often used to try and maintain sinus rhythm and prevent PAF. Periods of fast atrial fibrillation are commonly induced by trauma, anaesthesia and a stress response.

Those with new AF, persistent AF or PAF with a fast ventricular rate need review. Tachycardia may be due to pain, a cardiac event or sepsis and a 12-lead ECG, measurement of lactate and inflammatory markers is advised. Those with no evidence of inter-current illness may simply have new AF or poor rate control. Correction of dehydration and electrolyte imbalance should be initiated immediately. If the rate remains persistently above $110 \mathrm{bpm}$, then urgent rate control may be required preoperatively. Digoxin loading often takes $24 \mathrm{~h}$ to establish rate control. New prescription of betablockers is not advised pre-operatively due to concerns about hypotension. Short-acting intravenous metoprolol may be used with caution. The most effective method for rapid rate control is with intravenous amiodarone. This is usually administered with a slow bolus of $300 \mathrm{mg}$ over $1 \mathrm{~h}$ followed by a 24-h infusion of $0.5 \mathrm{mg} / \mathrm{kg} / \mathrm{h}$ (450 mg in $500 \mathrm{~mL}$ normal saline). This must be administered 
through a large bore cannula and ideally into a central line with cardiac monitoring. Cardiology advice may be required for complex patients.

\subsection{Management of Anticoagulants and Anti-platelets}

Anti-platelets are mainly used for secondary prevention of stroke, in peripheral vascular disease and following cardiac events. Anti-platelet agents cause irreversible platelet dysfunction and recovery only occurs with production of new platelets over 7-10 days. However, delay for urgent surgery, such as fractured neck of femur fixation, is not recommended [12]. With regard to choice of anaesthesia, aspirin is usually of little consequence, and SIGN guidance supports central neuraxial blockade in aspirin monotherapy [12]. Clopidogrel monotherapy should not delay surgery [13] and indeed should not be a contraindication to spinal or epidural anaesthesia, with little evidence for increase in rates of vertebral canal haematoma [12]. General anaesthesia should be considered for patients taking dual anti-platelet therapy [12]. Prophylactic peri-operative platelet transfusions are not necessary and should only be considered if there is excessive surgical bleeding [12].

Up to $40 \%$ of patients admitted with a hip fracture will be anticoagulated [14]. Direct oral anticoagulants (DOACs), such as apixaban, rivaroxaban, edoxaban and dabigatran, are currently prescribed more commonly than warfarin in the United Kingdom [15]. They have the advantage of fewer drug interactions, do not require plasma level monitoring for dosing and are effective and relatively safe [16].

In patients taking warfarin, AAGBI guidance recommends proceeding with surgery under general anaesthesia when the INR has been reduced by intravenous vitamin $\mathrm{K}$ to less than 2.0 [17]. Spinal anaesthesia and surgery are considered safe with an INR $<1.5$ [17].

Guidance for anaesthesia and surgery on patients with a hip fracture receiving DOACs is currently lacking. European and Scandinavian guidelines have advocated a pragmatic pharmacokinetic model with the passage of two half-lives between drug discontinuation and central neuraxial blockade [17].

The factor Xa inhibitors apixaban and rivaroxaban have a reversal agent, andexanet alfa, but it is only licenced for life-threatening bleeding and not routine use. The ANNEXA-4 study demonstrated effective clinical haemostasis with andexanet alfa, but thrombotic events occurred in $18 \%$ of patients during the 30-day follow-up [18].

Dabigatran, the direct thrombin inhibitor, has a licensed safe reversal agent, idarucizumab.

Traditional coagulation tests such as PT/INR and APTT are not sensitive in monitoring DOAC plasma activity [17]. Furthermore, the INR can be normal when clinically relevant plasma levels of DOAC are present. Therefore, traditional coagulation tests are not recommended. Plasma Xa assays are accurate but not commonly available in many hospital laboratories, and without an evidence base to guide the correlation of plasma levels to neuraxial performance safety, their use is limited. Conversely, clinical activity of dabigatran can be monitored easily and reliably through plasma thrombin time. 
With the secondary analysis of the national audit project ASAP 1 showing no difference in 30-day mortality between patients who received a general anaesthetic versus a spinal anaesthetic for hip fracture surgery, for many patients general anaesthesia is an acceptable alternative to spinal anaesthesia [19]. However, this is not a straightforward decision and should be made on an individual basis depending upon type of anticoagulant, renal function, the type of surgery required, anticipated blood loss, pain control and risk of immobility.

Table 7.2 gives details of suggested management for different anticoagulant medications.

Table 7.2 Anti-platelets and anticoagulants in patients with fragility fracture

\begin{tabular}{|c|c|c|c|}
\hline Drug & $\begin{array}{l}\text { Elimination } \\
\text { half-life }\end{array}$ & Management & $\begin{array}{l}\text { Acceptable to proceed } \\
\text { with spinal }\end{array}$ \\
\hline Warfarin & 4-5 days & $\begin{array}{l}5 \mathrm{mg} \text { vitamin } \mathrm{K} \text { intravenously } \\
\text { and repeat INR after } 4-6 \mathrm{~h} \text {. } \\
\text { This can be repeated or } \\
\text { consider Beriplex for } \\
\text { immediate reversal }\end{array}$ & If INR $<1.5$ \\
\hline Clopidogrel & $\begin{array}{l}\text { Irreversible } \\
\text { effect on } \\
\text { platelets }\end{array}$ & $\begin{array}{l}\text { Proceed with surgery } \\
\text { Monitor for blood loss } \\
\text { Consider platelet transfusion if } \\
\text { concerns regarding bleeding }\end{array}$ & $\begin{array}{l}\text { If anti-platelet } \\
\text { monotherapy. GA if } \\
\text { dual therapy }\end{array}$ \\
\hline $\begin{array}{l}\text { Unfractionated iv } \\
\text { heparin }\end{array}$ & $1-2 \mathrm{~h}$ & Stop iv heparin 2-4 h pre-op & $4 \mathrm{~h}$ \\
\hline $\begin{array}{l}\text { Low-molecular weight } \\
\text { heparin sub-cutaneous } \\
\text { prophylactic dose }\end{array}$ & $3-7 \mathrm{~h}$ & Last dose $12 \mathrm{~h}$ pre-op & $12 \mathrm{~h}$ \\
\hline $\begin{array}{l}\text { Low-molecular weight } \\
\text { heparin sub-cutaneous } \\
\text { Treatment dose }\end{array}$ & $3-7 \mathrm{~h}$ & $\begin{array}{l}\text { Last dose } 12-24 \mathrm{~h} \text { pre-op. } \\
\text { Monitor for blood loss }\end{array}$ & $24 \mathrm{~h}$ \\
\hline Ticagrelor & $8-12 \mathrm{~h}$ & $\begin{array}{l}\text { Proceed with surgery with } \\
\text { general anaesthetic } \\
\text { Monitor for blood loss } \\
\text { Consider platelet transfusion if } \\
\text { concerns regarding bleeding }\end{array}$ & $\begin{array}{l}5 \text { days or post platelet } \\
\text { transfusion at least } \\
6 \mathrm{~h} \text { post last dose }\end{array}$ \\
\hline Aspirin & $\begin{array}{l}\text { Irreversible } \\
\text { effect on } \\
\text { platelets }\end{array}$ & Proceed with surgery & Continue \\
\hline Rivaroxiban & $7-10 \mathrm{~h}$ & $\begin{array}{l}\text { Surgery and anaesthesia } 24 \mathrm{~h} \\
\text { after last dose if renal function } \\
\text { normal }\end{array}$ & $\begin{array}{l}2 \text { half-lives } / 24 \mathrm{~h} \text { after } \\
\text { last dose if renal } \\
\text { function normal }\end{array}$ \\
\hline Dabigatran & $12-24 \mathrm{~h}$ & $\begin{array}{l}\text { Surgery and anaesthesia if } \\
\text { thrombin time normal } \\
\text { or idarucizumab for immediate } \\
\text { reversal if thrombin time } \\
\text { prolonged }\end{array}$ & $\begin{array}{l}\text { If thrombin time } \\
\text { normal or } 30 \mathrm{~min} \\
\text { following } \\
\text { idarucizumab } \\
\text { infusion }\end{array}$ \\
\hline Apixiban & $12 \mathrm{~h}$ & $\begin{array}{l}\text { Surgery and anaesthesia } 24 \mathrm{~h} \\
\text { after last dose if renal function } \\
\text { normal }\end{array}$ & $\begin{array}{l}2 \text { half-lives } / 24 \mathrm{~h} \text { after } \\
\text { last dose if renal } \\
\text { function normal }\end{array}$ \\
\hline
\end{tabular}


Understanding the reason for anti-platelet/anticoagulant medication is essential in managing peri-operative risk of thromboembolic events. Patients with cardiac stents are at high risk of thrombosis and cardiac events and anti-platelet medication should either continue or be stopped for the shortest possible time.

Patients with mechanical heart valves (particularly mitral valves), known AF with recent stroke and recent DVT or PE are at high risk of peri-operative thromboembolic complications and bridging strategies should be considered. Treatmentdose subcutaneous low-molecular-weight heparin can be given until $24 \mathrm{~h}$ before surgery or intravenous unfractionated heparin until $2-4 \mathrm{~h}$ before surgery. The latter requires careful monitoring with 4-6 hourly APTT levels to ensure correct dosing.

Temporary insertion of an inferior vena cava filter should be considered for those with recent proximal DVT or PE.

Tranexamic acid has been shown to reduce the need for transfusion in a small study of patients with hip fracture with no difference in 3-month mortality [20], but in a similar small study, there appeared to be a significant increased risk of thromboembolic events [21]. A meta-analysis involving almost 600 patients suggested that Tranexamic acid administration reduces blood loss and transfusion rates with no significant difference in thrombotic events. The authors recommended a large, high-quality randomised control study to ensure safety and to establish clarity regarding the optimal regimen, dosage and timing before wide recommendation for use in hip fracture surgery [22].

\subsection{Anaemia}

Anaemia on admission is an independent predictor of poor outcome and is present in about $10-12 \%$ of those presenting with hip fracture [23]. It often reflects underlying disease such as malignancy, chronic kidney disease or poor nutrition. It is important to send blood for haematinics pre-transfusion to aid diagnosis and subsequent management. Macrocytic anaemia should not be transfused without an understanding of the cause and in liaison with haematologists. Although the evidence is controversial, most clinicians would aim for a pre-operative haemoglobin of at least $100 \mathrm{~g} / \mathrm{dL}$.

It is possible to predict blood loss depending upon the type of fracture with intracapsular fractures losing about $1000 \mathrm{~mL}$, extracapsular about $1200 \mathrm{~mL}$ and intertrochanteric or subtrochanteric up to $1600 \mathrm{~mL}$ [24]. This may be greater in those on anti-platelet therapy or anti-coagulants.

The FOCUS study was a large randomised controlled trial comparing liberal transfusion with restrictive transfusion in patients following hip fracture which showed no difference in mortality, ability to walk across a room at 60 days or length of hospital stay [25]. However, a decision about transfusion trigger should be made on an individual basis pre-operatively, taking into account frailty, cardiorespiratory reserve and levels of function. Usual practice is to keep haemoglobin above $80 \mathrm{~g} / \mathrm{dL}$ for those who are well and to aim for a haemoglobin of above $100 \mathrm{~g} / \mathrm{dL}$ for those with poor cardiorespiratory reserve. 
Intravenous iron pre-operatively has been evaluated but its effects are not sufficient or quick enough to reduce the need for blood transfusion in the first week after surgery [26].

\subsection{Diabetes}

Poor glycaemic control in the peri-operative period can lead to dehydration and poor wound healing with prolonged hyperglycaemia. Hypoglycaemia can also have serious consequences contributing to delirium, falls and seizures.

In the pre-operative period, patients with fragility fracture are often reluctant to eat due to pain, immobility and side effects of analgesia. Immobility may lead to reduced calorie requirements but pain and stress result in hyperglycaemia.

It is important to review diabetes medication pre-operatively and to monitor the blood sugar levels regularly. The AAGBI have produced comprehensive guidelines for peri-operative management of diabetes [27]. Patients who have taken longacting oral hypoglycaemics or long-acting insulin need close monitoring and may need slow $5 \%$ glucose infusion if being kept nil by mouth for surgery.

Pre-operative carbohydrate loading or high-sugar dietary supplements should be withheld in patients with diabetes as these may lead to poorly controlled blood sugar levels.

Most patients on oral hypoglycaemics can be managed by simply omitting usual medication on the day of surgery (NB: there is no need to stop pioglitazone). Metformin should be withheld for $48 \mathrm{~h}$ in anyone at risk of renal impairment, as there is an association with lactic acidosis. If pre-operative blood sugars rise above $12 \mathrm{mmol} / \mathrm{L}$, consider variable-rate intravenous insulin infusion (VRIII). Oral medication should restart as soon as the patient is able to eat and drink.

Those usually on short-acting or combined insulin preparations should omit their usual insulin dose and start on VRIII pre-operatively with intravenous fluid. This should be $5 \%$ glucose if the blood sugars are low. For patients with type I diabetes, insulin should never be stopped completely.

Long-acting insulin analogues (Glargine, Lantus, Detemir or Levimir) can be continued in the peri-operative period, with some people advocating reducing the dose by one-third.

It is important to make a post-operative plan and to withdraw the VRIII as soon as the patient is eating and drinking, to avoid fluid overload and electrolyte disturbance. Normal insulin doses may need adjusting until the patient is eating, drinking and mobilising normally.

\subsection{Chronic Kidney Disease (CKD)}

CKD is common in older people and can be associated with excess surgical morbidity [28]. It is important to establish the duration of CKD and baseline renal function. CKD may reflect impaired excretory function with raised urea, creatinine and 
metabolic products. In addition, there may be impaired synthetic function resulting in acidosis, hyperkalaemia, hypertension and oedema. CKD also results in reduced erythropoietin with anaemia and reduced hydroxylation of vitamin D causing hypocalcaemia and hyperphosphatemia. Platelet dysfunction is common in CKD, increasing the risk of bleeding.

Anaemia and metabolic abnormalities should be corrected to acceptable limits pre-operatively. Fluid overload is difficult to correct pre-operatively but those with end stage renal disease who are dialysis dependent should be dialysed within $24 \mathrm{~h}$ pre-operatively to reduce fluid overload.

Many drugs are excreted by the kidneys and can accumulate in patients with CKD. These may require dose adjustment or administration interval adjustment and, in some cases, avoided completely.

Anaesthesia often results in hypotension and a significant reduction in renal blood flow with worsening of renal function in the post-operative period. It is essential that anaesthetists are aware of patients with CKD who have poor renal reserve so that they can make every effort to prevent hypotension.

Patients with CKD often have concomitant ischaemic heart disease and continuation of beta blockers and correction of anaemia may help to reduce the incidence of cardiovascular events.

\subsection{Respiratory Disease}

Predicting those who are at highest risk of post-operative complications enables pre-operative intervention and optimisation. All patients with hip fracture are at risk of atelectasis and of chest infection which is one of the reasons for early operation and mobilisation. Those with underlying lung disease or smokers with undiagnosed lung disease have a higher risk. Low serum albumin, recent weight loss and dependency are also associated with increased risk [29].

Opiate analgesics and anaesthetic agents can reduce respiratory drive resulting in hypoxia, hypercapnia and atelectasis and should be used with caution.

Obesity also contributes to reduced gas exchange through reduced lung volume and in severe cases can lead to hypercapnic respiratory failure, but there is no evidence that patients with hip fracture and a high BMI have an increased rate of postoperative complications [30].

Cor pulmonale and pulmonary hypertension carry significant morbidity and mortality.

Pre-operative clinical assessment, chest X-ray and arterial blood gases give important baseline information.

Exacerbations of chronic obstructive airways disease may need treatment and optimisation pre-operatively, but most respiratory infections should not delay operation unless accompanied by sepsis, cardiovascular compromise or very high oxygen requirements.

The choice of anaesthetic is discussed in Chap. 8. 


\subsection{Medication Review}

In some countries, medicine reconciliation soon after admission is undertaken by a pharmacist. Understanding how a patient manages a complex regime is important, giving insight into cognition and compliance. Specific medication may suggest certain diagnoses, but care should be taken in making assumptions.

All regular medication should be written up on the drug chart with the indication for each drug and clear documentation of which should be continued or withheld pre-operatively. Most older patients with frailty admitted with fragility fracture will be volume depleted, and it is important to withhold medications which could contribute to renal hypoperfusion and acute kidney failure in the peri-operative period (e.g. diuretics, ACE inhibitors, anti-hypertensives).

Long-term sedatives (e.g. benzodiazepines, antipsychotics) should be reviewed and possibly reduced in the immediate peri-operative period, as many of the anaesthetic drugs will also cause sedation. However, they should not be stopped abruptly or withheld for prolonged periods of time.

Other medications must be given on the morning of surgery with a small sip of water (e.g. beta blockers for angina or rate control, anticonvulsants and medication for Parkinson's disease).

Some medications need reviewing and adjusting during the peri-operative period (see anticoagulants and anti-platelets and management of diabetes). Patients on hydrocortisone for pituitary failure or long-term low-dose steroid with possible adrenal failure should be given an increased dose usually $50 \mathrm{mg}$ of hydrocortisone on induction via intramuscular or intravenous route and three times a day for the first $24 \mathrm{~h}$ ). Inhalers may be changed to nebulisers for better delivery while a patient is immobile in bed.

Every prescribed medication should have a clear ongoing indication and benefits of the medication should outweigh the risks. Hospital admission with multidisciplinary input is an opportunity to review this. It is an important aspect of comprehensive geriatric assessment and takes considerable time. It should start pre-operatively but will need to continue to be reviewed and adjusted in the postoperative period.

Considerable thought should be given to medication that may contribute to falls (see Chap. 16).

\subsection{Preventing Complications: Thromboembolic Events}

Patients with fragility fracture are considered at particularly high risk of thromboembolic events, due to the effects of trauma, surgery and immobility. Older patients with frailty may have other co-morbidities such as heart failure or a history of thromboembolic events that increase this risk further. UK NICE guidelines recommend daily prophylactic-dose low-molecular-weight heparin (LMWH) for all hospitalised patients unless there are specific contraindications [31]. LMWH should be 
prescribed on admission but omitted if the patient is going to surgery within $12 \mathrm{~h}$. If there is likely to be a delay to surgery, pre-operative dosing should be considered taking into account risks of bleeding further into the fracture site.

The incidence of symptomatic venous thromboembolic events (VTE) is between $1 \%$ and $9 \%$ and symptomatic pulmonary emboli (PE) $0.2-1.7 \%$ following hip fracture surgery. However, the risk of significant bleeding with LMWH is $0.8-4.7 \%$ [32].

There is no good evidence for compression stockings in patients following hip fracture, and the potential harm in patients with poor skin and circulation should not be underestimated. Local policies should be followed, but with a review of risks and benefits in each individual patient.

\subsection{Antibiotic Prophylaxis}

Antibiotic prophylaxis is strongly recommended for surgical management of fractures to help to prevent deep wound infection. Each hospital will have its own policy to reflect likely pathogens and local patterns of resistance. This usually involves a single dose pre-operatively and 24-h cover post-operatively. Antibiotic choice may vary for patients who have fallen and fractured while in hospital or from a nursing home environment where the incidence of drug resistance is higher.

\subsection{Appropriate Ceilings of Care}

Many patients with fragility fracture have significant frailty and a quarter are in their last year of life. It is important that they and their next of kin have a realistic understanding of which treatments may result in benefit and which are likely to cause harm or distress. Organ failure as a result of end-stage chronic disease is usually irreversible, and under these circumstances organ support in an intensive care unit setting is likely to be ineffective and therefore inappropriate. Where there is a reversible element to organ failure, decisions regarding invasive treatments should be discussed in anticipation pre-operatively where possible.

Cardiopulmonary resuscitation in the event of cardiac arrest is unlikely to be effective in those with poor physiological reserve and an anticipatory form (Do Not Attempt Cardiopulmonary Resuscitation or DNACPR form) is required in some countries.

Many older people do not wish to receive life-prolonging treatments and may have discussed this with relatives or completed an advanced care plan. It is important to discuss this with the patient and their next of kin during the pre-operative assessment to ensure that all are aware of the patient's priorities. A DNACPR order may be reversed in the immediate peri-operative period, in theatre and in recovery area to ensure that recovery from anaesthesia is complete and does not contribute to cardiac or respiratory compromise. The use of drugs and techniques often used as part of CPR may be indicated in the short-term [33]. 
Table 7.3 Reasons for delaying surgery for hip fracture that the AAGBI working party considers unacceptable and acceptable [33]

\begin{tabular}{l|l}
\hline $\begin{array}{l}\text { Acceptable } \\
\text { Haemoglobin concentration }<8 \mathrm{~g} \mathrm{dL}^{-1}\end{array}$ & $\begin{array}{l}\text { Unacceptable } \\
\text { Lack of facilities or theatre } \\
\text { space }\end{array}$ \\
$\begin{array}{l}\text { Plasma sodium concentration }<120 \text { or }>150 \mathrm{mmol} \mathrm{L}^{-1} \\
\text { Potassium concentration }<2.8 \text { or }>6.0 \mathrm{mmol} \mathrm{L}^{-1}\end{array}$ & $\begin{array}{l}\text { Unavailable surgical expertise } \\
\text { Minor electrolyte abnormalities }\end{array}$ \\
\hline $\begin{array}{l}\text { Uncontrolled diabetes } \\
\text { Uncontrolled or acute onset left ventricular failure } \\
\text { Correctable cardiac arrhythmia with a ventricular rate } \\
>120 \mathrm{~min}^{-1}\end{array}$ & \\
$\begin{array}{l}\text { Chest infection with sepsis } \\
\text { Reversible coagulopathy }\end{array}$ & \\
\hline
\end{tabular}

\subsection{Conclusion}

Pre-operative assessment of patients with fragility fracture requires skill, time and effort. It is best achieved through multi-disciplinary review and information gathering to provide a clear and accurate understanding of a patient's background. Many patients require adjustments to medications in peri-operative period and for some urgent optimisation is necessary. For the majority of patients, proceeding with surgery without delay is in their best interests, as most intercurrent conditions are not rapidly reversible and medical instability will progress with poorly controlled pain from the fracture and an inability to sit upright. The AAGBI have produced guidelines on acceptable reasons to delay surgery [34]—-see Table 7.3. Good pre-operative assessment includes shared decision-making with regard to the best form of management for that individual, taking into account the risks and benefits in addition to the patient's priorities. Evidence-based protocols and guidelines are important, but ultimately this process requires clinical judgement and should involve a senior and experienced team.

\section{References}

1. Welsh T, Gordon A, Gladman J (2014) Comprehensive geriatric assessment-a guide for the non specialist. Int J Clin Pract 68(3):290-293

2. Marcantonio E, Flacker J, Wright R et al (2001) Reducing delirium after hip fracture: a randomized trial. J Am Geriatr Soc 49(5):516-522

3. Kalisvaart K et al (2005) Haloperidol prophylaxis for elderly hip surgery patients at risk for delirium: a randomised placebo controlled trial. J Am Geriatr Soc 53(10):1658-1666

4. www.the4AT.com

5. Bellelli G et al (2014) Validation of the 4AT; a new instrument for rapid delirium screening: a study in 234 hospitalised older people. Age Ageing 43:496-502. https://doi.org/10.1093/ ageing/afu021

6. Jette M et al (1990) Metabolic equivalents (METS) in exercise testing, exercise prescription, and evaluation of functional capacity. Clin Cardiol 13:555-565 
7. Goldman L, Caldera DL, Nussbaum SR et al (1977) Multifactorial index of cardiac risk in noncardiac surgical procedures. N Engl J Med 297(9):845-850

8. Lee T, Marcantonio E, Mangione C et al (1999) Derivation and prospective validation of a simple index for prediction of cardiac risk of major noncardiac surgery. Circulation 100:1043-1049

9. Spurrier E, Wordsworth D, Martin S et al (2011) Troponin T in hip fracture patients: prognostic significance for mortality at one year. Hip Int 16:757

10. McBrien M, Heyburn G, Stevenson M et al (2009) Previously undiagnosed aortic stenosis revealed by auscultation in the hip fracture population- echocardiographic findings, management and outcome. Anaesthesia 64:863-870

11. MRHA (2006) Perioperative management pacemakers/ICDs March 2006

12. Scotttish Intercollegiate Guidelines Network (2009) Management of hip fracture in older people

13. Dolman B, Moppett I (2015) Is early hip fracture surgery safe for patients on clopidogrel? Systematic review, meta-analysis and met-regression. Injury 46:954-962

14. Schermann $\mathrm{H}$ et al (2019) Safety of urgent hip fracture surgery protocol under the influence of direct oral anticoagulation medications. Injury 50(2):398-402

15. Mullins B, Akehurst H, Slattery D et al (2018) Should surgery be delayed in patients taking direct oral anticoagulants who suffer a hip fracture? A retrospective, case-controlled observational study at a UK major trauma centre. BMJ Open 8(4):e020625

16. Benzon H, Avram M, Green D et al (2013) New oral anticoagulants and regional anaesthesia. BJA 111(S1):i96-i113

17. AAGBI Safety Guideline (2011) Management of proximal femoral fractures

18. Connolly S, Milling T, Eikleboom J et al (2016) Andexanet alfa for acute major bleeding associated with factor Xa inhibitors. N Engl J Med 375:1131-1141

19. White SM et al (2016) Secondary analysis of outcomes after 11,085 hip fracture operations from the prospective UK Anaesthesia Sprint Audit of Practice (ASAP-2). Anaesthesia 71(5):506-514

20. Lee $\mathrm{C}$ et al (2015) The efficacy of tranexamic acid in hip hemiarthroplasty surgery: an observational cohort study. Injury 46(10):1978-1982

21. Zufferey P et al (2010) Tranexamic acid in hip fracture surgery: a randomized controlled trial. Br J Anaesth 104:23-30

22. Zhang P, He J, Fang Y et al (2017) Efficacy and safety of intravenous tranexamic acid administration in patients undergoing hip fracture surgery for hemostasis: a meta-analysis. Medicine 96(21):e6940

23. White $\mathrm{S}$ et al (2014) Outcome by mode of anaesthesia for hip fracture surgery. An observational audit of 65,535 patients in a national dataset. Anaesthesia 69:224-230. https://doi. org/10.1111/anae.12542

24. Foss N, Kehlet H (2006) Hidden blood loss after surgery for hip fracture. J Bone Joint Surg 88-B:1053-1059

25. Carson J, Michael M, Terrin L, et al for the FOCUS investigators (2011) Liberal or restrictive transfusion in high-risk patients after hip surgery. N Engl J Med 365:2453-2462

26. Moppett I, Rowlands M, Mannings A et al (2019) The effect of intravenous iron on erythropoiesis in older people with hip fracture. Age Ageing 48(5):751-755. https://doi.org/10.1093/ ageing/afz049

27. Dhatariya1 K, Levy N, Kilvert A et al, for the Joint British Diabetes Societies (2010) Diabetes UK position statements and care recommendations NHS diabetes guideline for the perioperative management of the adult patient with diabetes. Diabet Med 29:420-433

28. Salifu M et al (2016) Peri-operative management of the patient with chronic renal failure. Medscape. http://emedicine.medscape.com/article/284555-overview\#showall. Accessed 6 Apr 2016

29. Arozullah A, Conde M, Lawrence V (2003) Preoperative evaluation for postoperative pulmonary complications. Med Clin North Am 87(1):153-173 
30. Batsis J et al (2009) Body mass index and risk of non-cardiac postoperative medical complications in elderly hip fracture patients: a population based study. J Hosp Med 4(8):E1-E9

31. NICE Clinical Guideline 92 (2010) Venous thromboembolism: reducing the risk. www.nice. org.uk/nicemedia/pdf/CG92NICEGuidelinePDF.pdf

32. Rosencher N, Vielpeau C, Emmerich J et al (2005) Venous thromboembolism and mortality after hip fracture surgery: the ESCORTE study. J Thromb Haemost 3(9):2006-2014

33. The Association of Anaesthetists of Great Britain and Ireland (2009) Do not attempt resuscitation decisions in the peri-operative period. www.aagbi.org/sites/default/files/dnar

34. Griffiths R, Alper J, Beckingsale A et al (2012) Management of proximal femoral fractures 2011 Association of Anaesthetists of Great Britain and Ireland. Anaesthesia 67(1):85-98

Open Access This book is licensed under the terms of the Creative Commons AttributionNonCommercial-NoDerivatives 4.0 International License (http://creativecommons.org/licenses/ by-nc-nd/4.0/), which permits any noncommercial use, sharing, distribution and reproduction in any medium or format, as long as you give appropriate credit to the original author(s) and the source, provide a link to the Creative Commons license and indicate if you modified the licensed material. You do not have permission under this license to share adapted material derived from this book or parts of it.

The images or other third party material in this book are included in the book's Creative Commons license, unless indicated otherwise in a credit line to the material. If material is not included in the book's Creative Commons license and your intended use is not permitted by statutory regulation or exceeds the permitted use, you will need to obtain permission directly from the copyright holder.

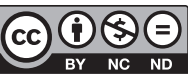

